\title{
The Mid-Term Outcome of Total Knee Arthroplasty in Patients With Prior High Tibial Osteotomy: A Prospective Study
}

\author{
Mahmoud Jabalameli, ${ }^{1}$ Mohammad Rahbar, ${ }^{1}$ Amin Moradi, ${ }^{2,}$ and Hoseinali $\mathrm{Hadi}^{3}$ \\ ${ }^{1}$ Bone and Joint Reconstruction Research Center, Shafa Orthopedic Hospital, Iran University of Medical Sciences, Tehran, IR Iran \\ ${ }^{2}$ Tabriz Shohada Hospital, Tabriz University of Medical Sciences, Tabriz, IR Iran \\ ${ }^{3}$ Arak University of Medical Sciences, Arak, IR Iran \\ "Corresponding author: Amin Moradi, Tabriz Shohada Hospital, Tabriz University of Medical Sciences, Tabriz, IR Iran. Tel: +98-9143102499, E-mail: moradi85ortho@yahoo.com
}

Received 2015 September 22; Revised 2016 April 29; Accepted 2016 May 27.

\begin{abstract}
Background: It is generally accepted that total knee arthroplasty (TKA) after high tibial osteotomy (HTO) is technically more difficult; however, there is still disagreement whether the subsequent arthroplasty is compromised.

Objectives: The current case series study aimed to assess patients treated by knee arthroplasty after a previous high tibial osteotomy and to determine the influence of this osteotomy on the clinical and radiologic outcomes of subsequent arthroplasty.

Methods: Up to April 2013, twenty-three patients with a history of twenty-five proximal tibial osteotomies prior to TKA were identified and compared with a matched group who underwent primary TKA in Shafa Orthopedic Hospital, Tehran, Iran. Demographic parameters of patients, surgical details, the knee range of motion (ROM), and American functional knee society (AKS) scores before and after TKA were recorded in the HTO and control groups. Weight bearing alignment radiographs were taken preoperatively and at the last follow-up. At the latest visit, patients' satisfaction was recorded.

Results: After an average of 49 months follow-up, all patients in the HTO and control groups were satisfied with their current level of function. In the HTO group, the rectus snip had to be used for better exposure in eight cases. There was no statistically significant difference between the two groups in postoperative knee ROM, mechanical leg alignment and mean functional knee society score, but the mean of operation time in the HTO group was significantly longer than that of the TKA time in the control group.

Conclusion: Although knee arthroplasty after HTO is technically more challenging than a primary procedure, the results of clinical scores, postoperative ROM and radiological evaluation in the study subjects were comparable with those of the primary TKA in midterm follow-up. Rectus snip is a safe procedure in such difficult cases for better exposure.
\end{abstract}

Keywords: Patient Outcome Assessment, Osteotomy, Arthroplasty, Knee

\section{Background}

High tibial osteotomy (HTO) is an established method to treat medial unicompartmental osteoarthritis (OA) of the varus knees in young and middle-aged patients (1-4). Rationale for the HTO is based on mechanical axis change of the lower limb, with load stress transfer from the pathological medial compartment to the still healthy lateral side $(2,5)$. However, the natural history of following HTO is more or less expected and with longer follow-up, the results worsen. Therefore, a high tibial osteotomy can be considered as a way to postpone total knee arthroplasty (TKA) in young patients (5-7). The results seem to deteriorate in time and overall failure rates of $20 \%$ at five years and $40 \%$ at eight years are reported $(8,9)$. Controversy exists regarding the success of a TKA after a previous $\operatorname{HTO}(7,8,10)$. Previous incisions, difficult exposure due to soft tissue scaring, patella baja, malunion or nonunion, offset of tibial shaft, change in the anatomy of tibial plateau, distortion of the joint line tilt and slope, retained osteosynthesis and suba- cute infections are technical challenges when performing a TKA after proximal tibia osteotomy (7-9). It is generally accepted that TKA after HTO is technically more difficult (10, 11); however, there is still disagreement whether the subsequent TKA is compromised. Although several comparative studies showed contradictory results; some (1, 2, 10, 12-14) suggesting that prior HTO does not change later TKA outcome and others $(5,11,15,16)$ concluding that the converse is true.

\section{Objectives}

The current case control study aimed to assess patients treated by knee arthroplasty after a previous HTO and determine the influence of HTO on the clinical and radiologic outcomes of the subsequent TKA and final extremity alignment. 


\section{Methods}

Before recruitment of the subjects, study protocol was approved by local ethics committee. The study was performed in accordance with the ethical standards of the 1964 Helsinki declaration. All patients signed the informed consent forms prior to recruitment in the study.

All patients who underwent TKA in Shafa orthopedic hospital, Tehran, Iran, were followed up prospectively since 2005. Up to April 2013, twenty-three patients with a history of twenty-five proximal tibial osteotomies prior to TKA were identified. Demographic parameters of patients, surgical details of high tibial osteotomy and TKA, the knees range of motion (ROM) before and after TKA were recorded. The closest living matched patients were selected from the same database to form the control group. The Patients were matched for gender, age, the knee ROM and length of follow up. The indication for TKA in all patients of the two groups was the same (symptomatic osteoarthritis) and one surgeon (senior) performed the reconstructive surgeries in both groups. Weight bearing alignment radiographs were taken preoperatively and at the last follow-up in both groups. At the latest visit, American knee society (AKS) scores were recorded. In addition, patients were asked whether they were satisfied with their operation or not (yes/no). The operating surgeons were not involved in the clinical evaluation. Further demographic, clinical and radiologic data are shown in Table1. There was no statistically significant difference between the two groups.

Table 1. Demographic and Preoperative Clinical and Radiologic Data

\begin{tabular}{|c|c|c|c|}
\hline & HTO Group & Control Group & P Value \\
\hline Number of patients & 23 & 25 & \\
\hline Number of knees & 25 & 25 & \\
\hline Mean age at TKA, $y$ & $63.3(5579)$ & $65(5281)$ & 0.52 \\
\hline Gender (male/female) & $4 / 19$ & $5 / 20$ & \\
\hline $\begin{array}{l}\text { Average Knee ROM, } \\
\text { degree }\end{array}$ & $106.5(70-130)$ & $110.1(75-130)$ & 0.23 \\
\hline $\begin{array}{l}\text { Mean of tibiofemoral } \\
\text { angle, degree }\end{array}$ & 7 varus $(-8-18)$ & 5.5 varus $(-3-11)$ & 0.14 \\
\hline $\begin{array}{l}\text { Mean of American knee } \\
\text { society score }\end{array}$ & $45.2(15-55)$ & $46.4(30-59)$ & 0.42 \\
\hline Average follow-up, mo & $49(25-90)$ & $48(24-88)$ & 0.66 \\
\hline
\end{tabular}

Abbreviations: HTO, high tibial osteotomy; ROM, range of motion; TKA, total knee arthroplasty.

In the HTO group, the average follow-up of the patients after TKA was 49 months and the average interval between HTO and TKA was 8.5 years. The tibial osteotomy was performed using two different techniques: twelve cases using dome-shaped osteotomy and thirteen ones by closing wedge osteotomy. Transverse scar in front of the knee was observed in five cases and longitudinal scar was observed in the others. Fixation devices used to fix closing wedge of the HTO were staples in nine cases and plates in four cases. Two cases after dome shape HTO had nonunion in the osteotomy site. One patient had a history of infection in the osteotomy site five years ago, but erythrocyte sedimentation rate (ESR) and C-reactive protein (CRP) were normal in all cases before TKA. The average maximum extension of the knees in the HTO group was 6.5 degrees $\left(-5^{\circ}\right.$ to $\left.20^{\circ}\right)$ and average maximum flexion was $112^{\circ}\left(70^{\circ}\right.$ to $\left.130^{\circ}\right)$.

All statistical analyses were performed using SPSS version 19.0 for Windows (SPSS Inc., Chicago, IL, USA). Data are presented as mean $\pm S D$, frequencies and percentages. Analysis was performed by the $\mathrm{X}^{2}$ test and Mann-Whitney $\mathrm{U}$ test. The confidence interval was $95 \% ; \mathrm{P}<0.05$ was considered level of significance.

\section{Results}

In the HTO group, a standard medial parapatellar arthrotomy was enough for exposure in many cases, but the rectus snip had to be used for better exposure in eight cases (three cases with history of dome-shaped osteotomy and five cases with previous closing wedge osteotomy). There was no need to use tibial tubercle osteotomy in any cases. The incision in all cases was longitudinal on the most lateral scar. Transverse scars were crossed at nearly $90^{\circ}$ angle. Fixation devices used for HTO were removed in 13 cases during the arthroplasty procedure. The type of implanted prosthesis was posterior stabilized NexGen LPS (Zimmer Inc., USA). In four cases, a NexGen modular tibial component with intramedullary stem and metal wedge was used to fill the large bone defect secondary to the osteotomy or cross and fix the nonunion area.

In the control group, a standard medial parapatellar arthrotomy was enough for exposure in all cases and there was no need to use the rectus snip or tibial tubercle osteotomy in any cases. The type of implanted prosthesis was posterior stabilized NexGen LPS (Zimmer Inc, USA) in all cases of the control group.

At the latest follow-up, the patients were evaluated clinically and radiologically. The closure of the wounds was good in all cases of the HTO and control groups and there were no necrosis and complications in the skin around the knees. The mean postoperative ROM of the knees in the HTO group was $122^{\circ}\left(90^{\circ}\right.$ to $\left.135^{\circ}\right)$ with average maximum extension of 2 degrees $\left(0-5^{\circ}\right)$ and the average maximum flexion was $125^{\circ}\left(95^{\circ}-135^{\circ}\right)$, close to the postoperative knee ROM in the control group. Postoperative results of the two groups were summarized in Table 2. There was no statistically significant difference between the groups in the post- 
operative knee ROM, mechanical leg alignment and mean Knee Society score, but the mean operation time of the HTO group was significantly longer than that of the TKA in the control group.

All patients in the HTO and control groups were satisfied with their current level of function and did not wish to consider further surgery. The nonunion in tibial osteotomy area was healed due to intramedullary longstem of the prostheses in the two nonunion cases. None of the knees demonstrated progressive or symptomatic radiolucent lines with a width of $2 \mathrm{~mm}$ or more at any follow-up periods.

Table 2. Postoperative Clinical and Radiological Data

\begin{tabular}{lccc}
\hline & HTO Group & Control Group & P Value \\
\hline Wound problem & 0 & 0 & \\
Mean operation time, min & $94(65-125)$ & $75(60-90)$ & 0.009 \\
\hline Rectus snip, knees & 8 & 0 & 0.000 \\
Average knee ROM, degree & $122(90-135)$ & $126(90-140)$ & 0.12 \\
$\begin{array}{l}\text { Mean tibiofemoral angle, } \\
\text { degree }\end{array}$ & 2 varus $(-2-4)$ & 3 varus $(-2-5)$ & 0.43 \\
\hline Mean knee society score & $85.5(55-100)$ & $87(65-100)$ & 0.18 \\
\hline
\end{tabular}

Abbreviations: HTO, high tibial osteotomy; ROM, range of motion.

\section{Discussion}

Many patients with medial compartment osteoarthritis of the knee ultimately require a TKA. HTO is an acceptable alternative in young and middle-aged patients since it permits high activity levels and delays the need for arthroplasty $(17,18)$. It typically results in pain relief and improved knee function in $80 \%-90 \%$ of patients $(19,20)$. However the natural history of following HTO is more or less expected and with longer follow-up, the results worsen $(5,6)$. Therefore, a high tibial osteotomy can be considered as a way to buy time and delay the need for TKA in young patients (5-7). A meta-analysis of follow-up studies showed that HTO had an average probability of a good result in $60.3 \%$ of patients in short term follow-up (9). Based on the natural cause of the disease, it was likely that most of such patients gained from their operation to some extent, although the outcome was not as good as expected (9). Although the short-term follow-up success of HTO showed good clinical outcome, the results seem to gradually deteriorate over time (19). According to the demographic transition towards a higher average age in the Society, more patients may require TKA after failed osteotomy $(20,21)$.

It is obvious that TKA after HTO is technically more demanding than a primary procedure (10-12) because of soft tissue scarring, a short patellar tendon, reduction of tibia bone stock, previous implants, joint line and anatomy distortion of proximal tibia and nonunion $(8,12)$. However it is still controversial whether or not HTO can have any negative effects on the outcome of a subsequent TKA $(7,8$, 10). Some studies showed that results of TKA after high tibial osteotomy could be inferior than that of the primary arthroplasty $(5,11,15,16)$. In a comparative study, Ragab et al. concluded that TKA after closed-wedge HTO was a technically demanding procedure and the expected balancing problems and the abnormal anatomy could lead to a clinical outcome less favorable for patients with osteotomy (5). In another study, Haslam followed 51 patients who underwent TKA after failed HTO for a long term (mean 12.5 years). There were more patients in the osteotomy group with a poor result, significantly reduced flexion and higher reoperation rate (11). Karabatsos et al. compared a matched group undergone TKA with and without history of HTO. They concluded that TKA after HTO was a technically more challenging procedure than the primary TKA and the functional outcomes at a mean follow-up of five years after TKA in patients with a previous HTO tended to be inferior but the differences were not significant (15).

On the other hand, some recent studies revealed opposite results $(1,10,12-14)$. In a study by Meding et al. the long-term functional scores and survival of bilateral TKAs were compared in patients with a previous HTO in one of the knees. They observed no differences in the Knee Society function, radiographic and pain scores between the knees without and with previous HTO. Terminal extension and flexion, arc of motion and knee alignment were similar between the knees. Survival at 15 years was $100 \%$ for knees without previous HTO and 97\% for knees with previous HTO. They concluded that previous high tibial osteotomy does not influence the function or survival of a TKA in long term (10). Efe et al. in a similar study suggested that although patients with a previous HTO had more complications, no significant differences were noted compared with the control group (12). Hernigou et al. evaluated the effect of malalignment after HTO on the outcome of TKA. They revealed that patients with an overcorrected HTO were more likely to require a soft tissue release to balance the knee. However, with a posterior stabilized arthroplasty, the degree of deformity had no impact on the longevity of the TKA (14).

Analysis of a systematic review study suggested that osteotomy did not compromise subsequent knee replacement. However, the writers could not extract a solid clinical conclusion because of the overall low quality of evidence (1).

The current study confirmed that TKA after HTO is a technically demanding procedure; posterior stabilized 
type prosthesis (NexGen LPS) had to be used in all cases. The mean of operation time in the HTO group was significantly longer than that of the TKA in the control group. The first point to consider during TKA after osteotomy is the choice of approach regarding the presence of a previous skin incision, with the possibility of removing possible fixation devices. It is noteworthy that no complication was observed in the skin of operation site. After skin incision, deep approach and exposure was the second challenge. Although in the control group, a standard medial parapatellar approach was enough for exposure, rectus snip had to be used for better knee exposure in eight cases of the HTO group. It reveals that previous HTO makes the knee exposure more difficult than the primary TKA. On the other hand, rectus snip does not change the standard TKA rehabilitation program.

In the cohort study by Amendola et al. the results of arthroplasty with previous HTO were compared with those of the primary TKA. Before arthroplasty, the AKS scores in the case and control groups were 47 and 45, respectively. After arthroplasty this score rose to 89.8 and 84 in the revision and control groups, respectively. Mean flexion at the last follow-up was $103^{\circ}$ (range $80^{\circ}-120^{\circ}$ ) in the case and $110^{\circ}$ in the control group (2). In the current study, the average maximum flexion was $125^{\circ}\left(95^{\circ}-135^{\circ}\right)$; better than that of their control group.

In the paper by Bae et al. it was recommended that the previous HTO itself had no deleterious effect on the outcome of the subsequent TKA. After mid- term follow-up of the 16 cases, they concluded that a meticulous surgical technique could produce satisfactory results in TKA after HTO; considering the correction of the deformity and the amount of the tibial bone resection. At the last visit, fourteen and two cases were excellent and good, respectively. The range of motion increased from $106.3^{\circ}$, before TKA, to $127.5^{\circ}$ at the last follow-up. They had good radiological outcomes and excellent postoperative alignment, without any progressive or significant radiolucency in the follow-up $\mathrm{X}$ rays (22).

The current study results were excellent in mid-term follow-up, similar to the above study. The mean ROM of the knees after TKA was $122^{\circ}$ and mean functional Knee Society score was 85.5 , close to the results of the control group (Table 2). All the patients were satisfied with their current level of function and did not wish to consider further surgery. In the radiological outcome, there was no significant difference between the final mean tibiofemoral angle of HTO and that of the control group; there was also no significant and symptomatic radiolucency in the follow-up radiographic exams. Good functional Knee Society score and acceptable ROM after TKA in the HTO group revealed that rectus snip is a safe procedure in these difficult cases for better exposure.

It seems that the results of the arthroplasty were gradually improved after HTO. Most of the studies demonstrating inferior results in the arthroplasties after HTO were published before $2007(5,11,15,16)$. But almost all the recent researches show that previous HTO does not have significant effects on the mid-term clinical and radiologic outcomes $(12,14,20,22)$. It is possible due to improvement in surgical techniques and the design of new prosthesis.

In summery, most data in the previous literatures as well as the present study fail to detect considerable negative effects of HTO on the later TKA outcomes. The current study had some limitations. The number of patients was small, and the follow-up period was relatively short with an average of 4.1 years. However, the patients under study had acceptable results at a mid-term follow-up. Welldesigned studies should investigate larger number of subjects to generate higher quality of evidence. Furthermore, long-term results are needed to reach more solid conclusions. Medial open-wedge high tibial osteotomy is a relatively new surgical approach to symptomatic varus knee and the cases of medial open-wedge HTO that need TKA are rare. In future, some studies should evaluate the problems of TKA in such cases. Although knee arthroplasty after HTO is technically more challenging than a primary procedure and needs to consider many details, the results of clinical scores, postoperative range of motion of the knee and radiological evaluation in these cases were comparable with those of the primary TKA in mid-term follow-up. Furthermore, it seems that rectus snip is a safe procedure in such difficult cases for better exposure. High quality observational studies with long term follow-up are needed to produce larger numbers and potentially generate higher quality of evidence to reach more solid conclusions.

\section{Footnote}

Author's Contribution: Design and supervision: Mahmoud Jabalameli; drafting, data analysis, writing and critical revision of the manuscript for important intellectual content: Mahmoud Jabalameli, Mohammad Rahbar, Amin Moradi and Hoseinali Hadi.

\section{References}

1. van Raaij TM, Reijman M, Furlan AD, Verhaar JA. Total knee arthroplasty after high tibial osteotomy. A systematic review. BMC Musculoskelet Disord. 2009;10:88. doi: 10.1186/1471-2474-10-88. [PubMed: 19619275].

2. Amendola L, Fosco M, Cenni E, Tigani D. Knee joint arthroplasty after tibial osteotomy. Int Orthop. 2010;34(2):289-95. doi: 10.1007/s00264009-0894-y. [PubMed: 19882155]. 
3. Marcacci M, Iacono F, Zaffagnini S, Marchetti PG. Total knee arthroplasty after proximal tibial osteotomy. Chir Organi Mov. 1995;80(4):353-9. [PubMed: 8706541].

4. Lee SC, Jung KA, Nam CH, Jung SH, Hwang SH. The short-term followup results of open wedge high tibial osteotomy with using an Aescula open wedge plate and an allogenic bone graft: the minimum 1-year follow-up results. Clin Orthop Surg. 2010;2(1):47-54. doi: 10.4055/cios.2010.2.1.47. [PubMed: 20191001].

5. Ragab RK, El-Sayed AMM. Total knee arthroplasty after high tibial osteotomy. A comparative study. Bull Alex Fac Med. 2008;44(4).

6. Meding JB, Keating EM, Ritter MA, Faris PM. Total knee arthroplasty after high tibial osteotomy. A comparison study in patients who had bilateral total knee replacement.JBone Joint Surg Am. 2000;82(9):1252-9. [PubMed: 11005516].

7. van Raaij TM, Bakker W, Reijman M, Verhaar JA. The effect of high tibia osteotomy on the results of total knee arthroplasty: a matched case control study. BMC Musculoskelet Disord. 2007;8:74. doi: 10.1186/14712474-8-74. [PubMed: 17683549].

8. Jabalameli M. Total knee arthroplasty after high tibial osteotomy. Shafa Orthoped J. 2013;1(1).

9. Virolainen P, Aro HT. High tibial osteotomy for the treatment of osteoarthritis of the knee: a review of the literature and a meta-analysis of follow-up studies. Arch Orthop Trauma Surg. 2004;124(4):258-61. doi 10.1007/s00402-003-0545-5. [PubMed: 12827394].

10. Meding JB, Wing JT, Ritter MA. Does high tibial osteotomy affect the success or survival of a total knee replacement?. Clin Orthop Relat Res. 2011;469(7):1991-4. doi: 10.1007/s11999-011-1810-5. [PubMed: 21424244].

11. Haslam P, Armstrong M, Geutjens G, Wilton TJ. Total knee arthroplasty after failed high tibial osteotomy long-term followup of matched groups. I Arthroplasty. 2007;22(2):245-50. doi 10.1016/j.arth.2006.01.031. [PubMed: 17275642].

12. Efe T, Heyse T], Boese C, Timmesfeld N, Fuchs-Winkelmann S, Schmit J, et al. TKA following high tibial osteotomy versus primary TKA-a matched pair analysis. BMC Musculoskelet Disord. 2010;11:207. doi 10.1186/1471-2474-11-207. [PubMed: 20840744].

13. Kazakos KJ, Chatzipapas C, Verettas D, Galanis V, Xarchas KC, Psillakis I. Mid-term results of total knee arthroplasty after high tib- ial osteotomy. Arch Orthop Trauma Surg. 2008;128(2):167-73. doi: 10.1007/s00402-007-0488-3. [PubMed: 18008079].

14. Hernigou P, Duffiet P, Julian D, Guissou I, Poignard A, FlouzatLachaniette $\mathrm{CH}$. Outcome of total knee arthroplasty after high tibial osteotomy: does malalignment jeopardize the results when using a posterior-stabilized arthroplasty?. HSS J. 2013;9(2):134-7. doi: 10.1007/s11420-013-9344-x. [PubMed: 24426858].

15. Karabatsos B, Mahomed NN, Maistrelli GL. Functional outcome of total knee arthroplasty after high tibial osteotomy. Can I Surg. 2002;45(2):116-9. [PubMed: 11939653].

16. Madan S, Ranjith RK, Fiddian NJ. Total knee replacement following high tibial osteotomy. Bull Hosp Jt Dis. 2002;61(1-2):5-10. [PubMed: 12828373].

17. Haddad FS, Bentley G. Total knee arthroplasty after high tibial osteotomy: a medium-term review. J Arthroplasty. 2000;15(5):597-603. [PubMed: 10959998].

18. Parvizi J, Hanssen AD, Spangehl MJ. Total knee arthroplasty following proximal tibial osteotomy: risk factors for failure. J Bone Joint Surg Am. 2004;86-A(3):474-9. [PubMed: 14996871].

19. Bastos Filho R, Magnussen RA, Duthon V, Demey G, Servien E, Granjeiro JM, et al. Total knee arthroplasty after high tibial osteotomy: a comparison of opening and closing wedge osteotomy. Int Orthop. 2013;37(3):427-31. doi:10.1007/s00264-012-1765-5. [PubMed: 23288047].

20. Akasaki Y, Matsuda S, Miura H, Okazaki K, Moro-oka TA, Mizuuchi $\mathrm{H}$, et al. Total knee arthroplasty following failed high tibial osteotomy: mid-term comparison of posterior cruciate-retaining versus posterior stabilized prosthesis. Knee Surg Sports Traumatol Arthrosc. 2009;17(7):795-9. doi: 10.1007/s00167-009-0790-4. [PubMed: 19357836].

21. Bathis H, Perlick L, Tingart M, Luring C, Perlick C, Grifka J. Flexion gap configuration in total knee arthroplasty following high tibial osteotomy. Int Orthop. 2004;28(6):366-9. doi:10.1007/s00264-004-05955. [PubMed: 15597173].

22. Bae DK, Song SJ, Yoon KH. Total knee arthroplasty following closed wedge high tibial osteotomy. Int Orthop. 2010;34(2):283-7. doi: 10.1007/s00264-009-0749-6. [PubMed: 19367405]. 